\title{
Caracterización de pacientes diabéticos en hemodiálisis en la región de Atacama. Chile
}

\author{
Alejandra González, Belén Cabezas, Abigail Canto \\ Facultad de Ciencias de la Salud. Departamento de Enfermería. Universidad de Atacama. Chile
}

Como citar este artículo:

González A, Cabezas B, Canto A. Caracterización de pacientes diabéticos en hemodiálisis en la región de Atacama. Chile. Enferm Nefrol. 2020 Ene-Mar;23(1):75-82

\section{Resumen}

Introducción: La diabetes es una de las principales causas de enfermedad renal crónica en Chile y el mundo, por lo que es fundamental la prevención del avance de la nefropatía diabética para evitar la evolución de la enfermedad renal crónica.

Objetivo: Determinar las características de pacientes diabéticos que iniciaron tratamiento renal sustitutivo con hemodiálisis debido a una nefropatía diabética.

Material y Método: Se realizó un estudio cuantitativo de tipo descriptivo, con pacientes diabéticos y en hemodiálisis, mediante aplicación del test de Morisky Green y un instrumento de valoración sociodemográfico. Los sujetos fueron seleccionados mediante muestreo intencionado, tras su consentimiento informado.

Resultados: Los sujetos estudiados en su mayoría fueron mujeres, con diabetes tipo 2 en edad adulta, de un estrato económico bajo, escolaridad básica y media, y pertenecientes al Sistema de Salud Público. Gran parte indicó que tenía 5 o más años con diabetes al momento de ingresar a diálisis. $71,4 \%$ de ellos no tuvo derivación a Nefrólogo y 57,1\% refirió no haber sido adherente a su tratamiento, previo ingreso a diálisis.

\section{Correspondencia:}

Alejandra González Vargas

E-mail: alejandra.gonzalez@uda.cl
Conclusiones: El perfil del paciente diabético que inicia HD de nuestro estudio, son en su mayoría mujeres de edad media, con diagnóstico previo de DM2 sobre la DM1, y con más de 20 años con el diagnóstico de DM. En cuanto al perfil socioeconómico predominan los pacientes con bajos ingresos económicos, estudios básicos y medios. Por último destacar que presentaron mejor adherencia al tratamiento los hombres respecto a las mujeres.

PALABRAS CLAVES: diabetes; diabetes mellitus tipo 2; complicaciones de la diabetes; diálisis renal.

\section{Characterization of diabetic patients in hemodialysis in the region of Atacama. Chile}

\section{Abstract}

Introduction: Diabetes is one of the main causes of chronic kidney disease in Chile and in the world. It is essential to prevent the progression of diabetic nephropathy to prevent the evolution of chronic kidney disease.

Objective: To determine the characteristics of diabetic patients who started renal replacement therapy with hemodialysis due to diabetic nephropathy.

Material and Method: A descriptive quantitative study was carried out, with diabetic patients and on hemodialysis, by applying the Morisky-Green test and a 
sociodemographic assessment instrument. The subjects were selected by intentional sampling, after their informed consent.

Results: The subjects studied were mostly women, with type 2 diabetes (T2D) in adulthood, with a low economic level, basic and intermediate level education, and belonging to the Public Health System. In most of the subjects it was indicated that they had 5 or more years with diabetes disease at the time of starting dialysis. $71.4 \%$ of them were not referred to the nephrologist and $57.1 \%$ said they had not adhered to the treatment, after starting dialysis.

Conclusions: The profile of the diabetic patient who begins on hemodialysis in our study is mostly middleaged women, with a higher previous diagnosis of T2D over T1D, and with more than 20 years with the diagnosis of DM. Regarding the socioeconomic profile, patients with low economic income and no training or basic studies predominate. Finally, men had better adherence to treatment than women.

KEYWORDS: diabetes; type 2 diabetes mellitus; complications of diabetes; renal dialysis.

\section{Introducción}

La diabetes mellitus (DM) es una enfermedad endocrino-metabólica caracterizada por elevados niveles de glucosa en sangre. Entre los tipos de diabetes se destacan, la diabetes mellitus tipol (DM1), la tipo2 $(D M 2)$ y la diabetes mellitus gestacional $(D M G)^{1}$. En año 2015 la International Diabetes Ferderation (IDF), reveló que existían 415 millones de adultos que la padecían y que había 318 millones de prediabéticos que la padecerían también. En América del Sur la realidad sería similar, siendo un total de 29,6 millones de personas diagnosticadas, con proyección de llegar a un total de 48,8 millones al año $2040^{2}$.

El año 2008 un estudio indicó la prevalencia de las complicaciones más communes de la DM2, presentando $27,8 \%$ enfermedad renal, $18,9 \%$ daño ocular y el $9 \%$ enfermedad coronaria ${ }^{3}$. Mientras que el riesgo de morir que tienen las personas con DM es mucho mayor que el de quienes no poseen la enfermedad ${ }^{4}$. Las personas con DM ven afectados sus años de productividad, a lo que se suman los costos económicos por la enfermedad ${ }^{5}$, tanto para quienes la padecen, como para los Estados, consumiendo entre 5 y $20 \%$ del presupuesto destinado a salud a nivel mundial ${ }^{3}$. Entre las complicaciones asociadas a la DM, destaca la Nefropatía Diabética (ND) dado que puede evolucionar a Enfermedad Renal Crónica (ERC), la cual actualmente se considera una pandemia que afecta aproximadamente al $10 \%$ de la población adulta en diferentes partes del mundo, de las cuales un $90 \%$ de las personas que la padecen no lo saben ${ }^{6}$.

La National Kidney Foundation (NFK) ha clasificado la ERC en cinco etapas, según su progresión, para lo cual se ha determinado que el volumen de filtrado glomerular (VFG) sea representativo de la funcionalidad de los riñones y sea la unidad de medida del daño renal. De este modo la etapa 1 considera daño renal con un VFG normal de 90 o más, disminuyendo hasta la etapa 5 con VFG menor a 157. La progresión de la ERC puede tomar varios años hasta la aparición de los primeros síntomas y diagnóstico de la enfermedad ${ }^{8}$, sin embargo, al ser diagnosticada a tiempo y tratarse adecuadamente la progresión de la ERC podría ser mucho menor. La etapa 5 ocurre cuando el riñón no puede eliminar los residuos metabólicos corporales y realizar sus funciones regulatorias ${ }^{9}$. Actualmente cerca de veintemil personas en Chile padecen ERC en etapa 5 , de las cuales aproximadamente un $45 \%$ es por causa de $\mathrm{DM}^{10}$. En dicha etapa el usuario requiere de una terapia de sustitución renal, entre ellas destaca la hemodiálisis (HD) y la diálisis peritoneal (DP), las que reemplazan la función excretora renal ${ }^{11}$.

La terapia de HD consiste en la depuración de la sangre del usuario al salir mediante un acceso vascular a un sistema de circulación extracorpóreo, pasando a través de un filtro que permite la eliminación de toxinas y agua en exceso, haciendo que retorne al usuario sangre limpia ${ }^{7,12,13}$.

En Chile los tratamientos de diálisis están cubiertos en su totalidad por las Garantías Explicitas en Salud (GES), lo cual significa que todos los pacientes tienen garantizado el acceso a tratamiento de forma gratuita. Sin embargo los costos son altos ${ }^{14}$, cerca del $30 \%$ del presupuesto de Salud en Chile, es destinado a financiar la ERC, la que incluye diálisis y trasplante renal ${ }^{7}$.

La evolución de los diabéticos con ND a ERC en etapa terminal, no es parte de una regla, existen muchos pacientes con ND que nunca llegan a presentar ERC, sin embargo un grupo no menor evoluciona con ERC en etapa terminal. 
El objetivo del presente trabajo ha sido determinar las características de pacientes diabéticos que iniciaron tratamiento renal sustitutivo con hemodiálisis debido a una nefropatía diabética, entre la población de Atacama (Chile).

\section{Material y Método}

\section{Diseño}

Estudio de tipo cuantitativo, descriptivo, de corte transversal con usuarios con DM, ND y tratamiento periódico de HD durante el período de abril a mayo de 2018. Se utilizó una estrategia de muestreo no probabilístico intencional.

\section{Ámbito de estudio}

El estudio se realizó en 3 centros de HD de la Región de Atacama, que accedieron a participar de la investigación: Centro Renacer en el Valle, Centro Diálisis Vallenar y centro de Diálisis de Diego de Almagro. Cabe destacar que el estudio tambien incluyó pacientes de la Unidad de HD del Hospital Provincial del Huasco, quienes en ese momento se encontraban realizando su terapia de HD en los centros antes mencionados por problemas en su unidad de diálisis correspondiente.

\section{Población y muestra}

La población estudiada estuvo formada por un total de 50 usuarios. Una vez aplicados los criterios de inclusión y exclusión, la muestra resultante fue de 42 pacientes.

\section{Criterios de inclusión y exclusión}

Los criterios de inclusión fueron pacientes mayores de 18 años, en tratamiento con HD debido a una ERC secundaria a ND. Como criterios de exclusión se tuvieron en cuenta: pacientes con sesiones transitorias de $\mathrm{HD}$; pacientes con deterioro cognitivo.

\section{Procedimiento de recopilación de datos}

Se realizó una solicitud formal por parte del Depatarmento de Enfermería de la Universidad de Atacama, para invitar a participar en la investigación a los centros de HD de la ciudad de Vallenar, Diego de Almagro y Copiapó, en donde los centros de diálisis de esta última ciudad no aceptaron. Posteriormente se realizó la coordinación con cada uno de los centros de diálisis. Las entrevistas fueron realizadas por estudiantes de Enfermería de la Universidad en un período de 2 meses (abril y mayo 2018). Los participantes fueron informados acerca de la investigación y firmaron voluntariamente el Consentimiento Informado.

\section{Instrumento}

El cuestionario que se utilizó para recopilar datos contenía dos secciones, una para la historia clínica e información sociodemográfica (sexo, edad, tipo de DM, nivel socioeconómico, previsión de salud, años de escolaridad, años con patología base, grupo étnico y derivación a nefrólogo previo al ingreso HD) y otra para la medición de la adherencia terapéutica mediante aplicación del Test de Morisky Green, cuyas preguntas se contestan mediante respuesta dicotómica ó mediante la elección de una categoría. El test de Morisky Green el cual se compone de 4 preguntas cerradas: (1) ¿이vida alguna vez tomar los medicamentos para tratar su enfermedad? (2) ¿Se olvida de tomar los medicamentos a las horas indicadas? (3) Cuando se encuentra bien, ¿Deja de tomar la medicación? (4) Si alguna vez le sienta mal, ¿Deja usted de tomarla?. Cada item debe ser respondido dicotomicamente, con un sí o un no. La respuesta de un sí o más, indica la no adherencia terapeutica.

\section{Consideraciones éticas}

Se contó con la aprobación de una junta de académicos del Departamento de Enfermería de la Universidad de Atacama y de cada uno de los centros de diálisis en que se aplicó el instrumento. También se obtuvo el consentimiento verbal y escrito de cada paciente antes de incluirlo en el estudio.

\section{Análisis estadístico}

Se realizó una base de datos, utilizando el programa Excel Office 2010 (Microsoft) para su tabulación, donde se dividió cada variable operacional. Se utilizó el programa como interfase estadístico SPSS de IBM, que permitió operacionalizar las variables del estudio para su posterior análisis. Los resultados fueron expuestos y analizados por medios de tablas y gráficos descriptivos de frecuencia, resguardando en todo momento la identificación de los participantes.

\section{Resultados}

Las características sociodemográficas de la muestra se reflejan en la Tabla 1. En su mayoría los participantes fueron mujeres representando el 59,5\%, las edades de los participantes oscilaron entre los 18 y 86 años, cuyo mayor porcentaje se encontró en el rango de edad entre 31 a 64 años (57,1\%). Destacó el diagnóstico previo 
Tabla 1. Variables socio demográficas.

\begin{tabular}{|c|c|c|c|}
\hline Variables & Categorías & Frecuencia & Porcentaje \\
\hline Sexo & $\begin{array}{l}\text { Mujer } \\
\text { Hombre }\end{array}$ & $\begin{array}{l}25 \\
17\end{array}$ & $\begin{array}{l}59,5 \% \\
40,5 \%\end{array}$ \\
\hline Edad & $\begin{array}{c}18-30 \text { años } \\
\text { 31-64 años } \\
65 \text { años y más }\end{array}$ & $\begin{array}{c}0 \\
24 \\
18\end{array}$ & $\begin{array}{c}0 \% \\
57,1 \% \\
42,9 \%\end{array}$ \\
\hline Tipo de DM & $\begin{array}{l}\text { DM Tipo } 1 \\
\text { DM Tipo } 2\end{array}$ & $\begin{array}{c}2 \\
40\end{array}$ & $\begin{array}{c}4,8 \% \\
95,2 \%\end{array}$ \\
\hline Nivel socioeconómico & $\begin{array}{l}\text { Quintil } 1 \\
\text { Quintil } 2 \\
\text { Quintil } 3 \\
\text { Quintil } 4 \\
\text { Quintil } 5\end{array}$ & $\begin{array}{c}1 \\
15 \\
5 \\
8 \\
13\end{array}$ & $\begin{array}{c}2,4 \% \\
35,7 \% \\
11,9 \% \\
19 \% \\
31 \%\end{array}$ \\
\hline Previsión de salud & $\begin{array}{c}\text { Fonasa A } \\
\text { Fonasa B } \\
\text { Fonasa C } \\
\text { Fonasa D } \\
\text { Isapre } \\
\text { Prais } \\
\text { Dipreca }\end{array}$ & $\begin{array}{c}16 \\
15 \\
2 \\
7 \\
1 \\
1 \\
0\end{array}$ & $\begin{array}{c}38,1 \% \\
35,7 \% \\
4,8 \% \\
16,7 \% \\
2,4 \% \\
2,4 \% \\
0 \%\end{array}$ \\
\hline Años de escolaridad & $\begin{array}{c}0 \text { a } 4 \text { años } \\
5 \text { a } 8 \text { años } \\
9 \text { a } 12 \text { años } \\
13 \text { años y más }\end{array}$ & $\begin{array}{c}9 \\
12 \\
16 \\
5\end{array}$ & $\begin{array}{l}21,4 \% \\
28,6 \% \\
38,1 \% \\
11,9 \%\end{array}$ \\
\hline Años con patología base & $\begin{array}{c}0 \text { a } 4 \text { años } \\
5 \text { a } 9 \text { años } \\
10 \text { a } 14 \text { años } \\
15 \text { a } 19 \text { años } \\
20 \text { a } 24 \text { años } \\
25 \text { años y más }\end{array}$ & $\begin{array}{c}1 \\
8 \\
7 \\
6 \\
10 \\
10\end{array}$ & $\begin{array}{c}2,4 \% \\
19 \% \\
16,7 \% \\
14,3 \% \\
23,8 \% \\
23,8 \%\end{array}$ \\
\hline Grupo étnico & $\begin{array}{l}\text { Diaguita } \\
\text { Aymara } \\
\text { Mapuche } \\
\text { Otros } \\
\text { Ninguno }\end{array}$ & $\begin{array}{c}5 \\
1 \\
3 \\
2 \\
31\end{array}$ & $\begin{array}{c}11,9 \% \\
2,4 \% \\
7,1 \% \\
4,8 \% \\
73,8 \%\end{array}$ \\
\hline Adherencia a tratamiento & $\begin{array}{l}\text { SÍ } \\
\text { NO }\end{array}$ & $\begin{array}{l}18 \\
24\end{array}$ & $\begin{array}{l}42,9 \% \\
57,1 \%\end{array}$ \\
\hline Derivación a nefrólogo & $\begin{array}{l}\text { SÍ } \\
\text { NO }\end{array}$ & $\begin{array}{l}12 \\
30\end{array}$ & $\begin{array}{l}28,6 \% \\
71,4 \%\end{array}$ \\
\hline
\end{tabular}

de DM 2 por sobre la DM 1. En cuanto a los ingresos económicos prevalece la categoría Quintil 2, con ingresos mensuales entre los $\$ 51.000$ - $\$ 116.000$ pesos. En cuanto al Sistema Previsional, predomina el Sistema de Salud Pública con las categorías de Fonasa A y Fonasa $B$, lo que se relaciona a los bajos ingresos percibidos. En cuanto a los años de estudio, el mayor porcentaje de usuarios ha estudiado entre de 9 y 12 años, correspondiendo al $38,1 \%$ de sujetos que han cursado educación media completa ó incompleta; seguido por el $28,6 \%$ que ha estudiado entre 5 y 8 años, habiendo cursado solo enseñanza básica. Respecto a los años de DM el mayor porcentaje de pacientes tienen más de 20 años con DM. Destacó que un gran porcentaje de la población total estudiada no fue adherente al tratamiento diabético previamente al ingreso $H D$, siendo más de la mitad de la muestra, representando el 57,1\%.

Un alto porcentaje de la población no fue derivada a nefrólogo previo al ingreso a HD, correspondiendo al $71,4 \%$, representando a más de la mitad del total de la muestra (Ver Tabla 1).

Se observó que el mayor porcentaje de mujeres que ingreso a HD secundaria a ND fueron aquellas diagnosticadas entre 5 a 9 años de DM (28\%) (Ver Gráfico 1). Mientras que el mayor porcentaje de la población de hombres que ingreso a HD producto de ND, tenían 25 o más años con su patología de base DM $(35,3 \%)$ (Ver Gráfico 2).

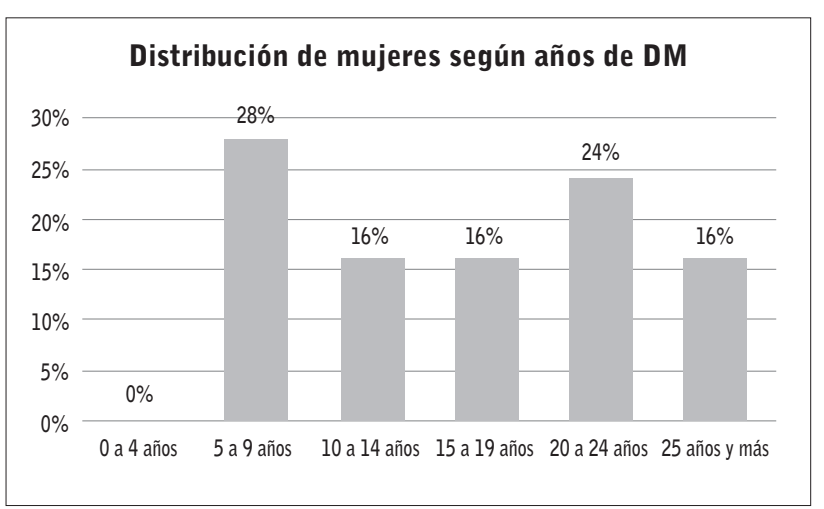

Gráfico 1. Distribucción de mujeres según años de Diabetes Mellitus (DM).

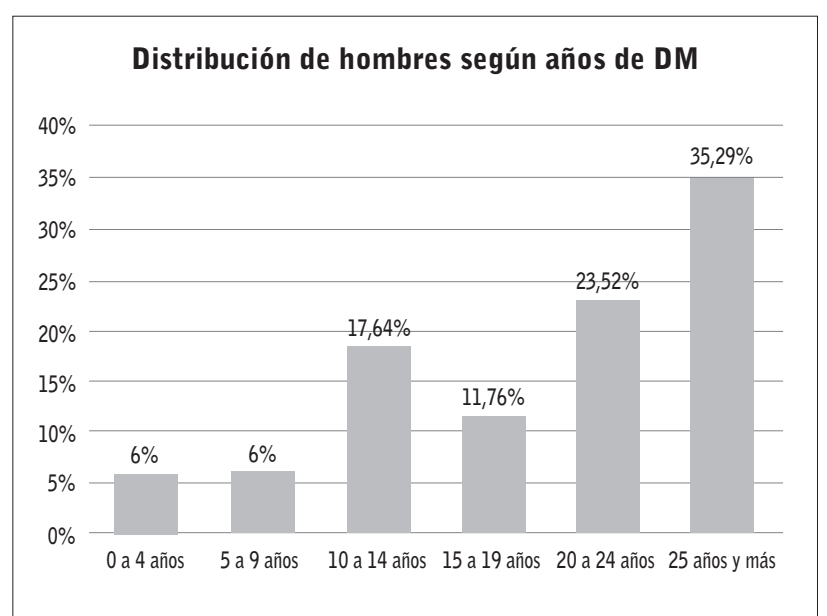

Gráfico 2. Distribucción de hombres según años de Diabetes Mellitus (DM). 
Por último, al comparar las variables sexo/adherencia al tratamiento se obtiene que en la población de hombres el mayor porcentaje de estos son adherentes con un $58,9 \%$. En cambio en población de mujeres el mayor porcentaje de estas son no adherentes al tratamiento siendo un $68 \%$ (Ver Gráfico 3).

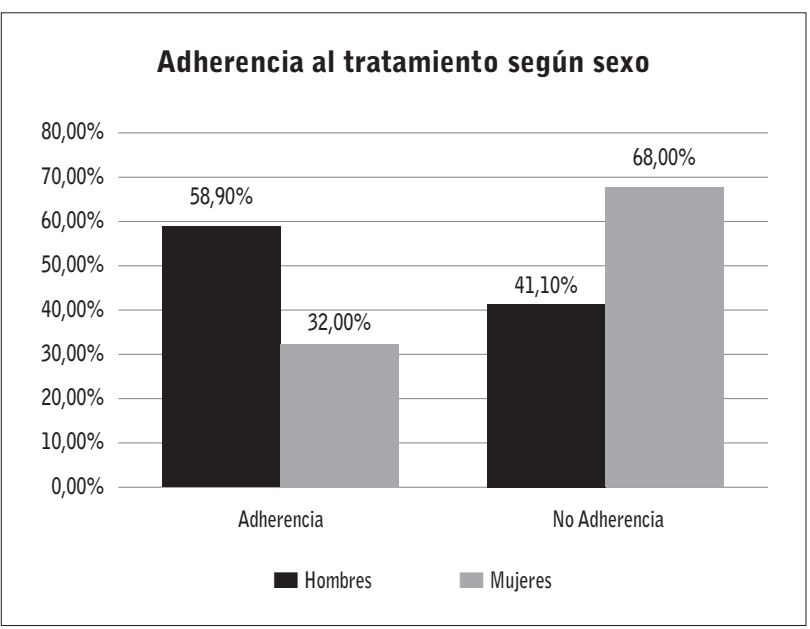

Gráfico 3. Población de mujeres y hombres, según adherencia - no adherencia al tratamiento.

\section{Discusión}

En cuanto a las características socio demográficas, el sexo más prevalente en ingresar a HD producto de ND son las mujeres, pudiendo relacionarse esta mayor incidencia femenina a la falta de autocuidado en la mujer a causa de su rol sociocultural como protectora del entorno. Un estudio realizado en Costa Rica concluyó: "Las acciones de las mujeres con respecto a su salud son un fiel reflejo de la construcción cultural de su identidad", "esta forma de aprender a estar en el mundo hace que no puedan generar cambios sustantivos dirigidos al cuidado de su salud"15. En cuanto al rango de edad, el mayor porcentaje estuvo entre los 31 a 64 años $(57,1 \%)$, similar a una investigación realizada en Ecuador la cual indica que la mayor cantidad de pacientes que desarrollan ND tienen una edad media de 64 años $^{16}$, sin embargo, a nivel nacional la mayor incidencia se da entre los 61 y 70 años. Pudiendo existir un ingreso más temprano a HD de los usuarios que ingresan a causa de ND, lo cual hace que el riesgo de morbimortalidad aumente a temprana edad.

El tipo de diabetes más predominante fue DM2 $(95,24 \%)$, resultado similar a la realidad del país, en donde del total de usuarios con Nefropatía basal a causa de $\mathrm{DM}(46,6 \%)$, el $40,4 \%$ es por $\mathrm{DM} 2^{10}$.

Con respecto al nivel socioeconómico la población estudiada se encontró en el Quintil 2 representando al $35,7 \%$ del total, siendo personas que pertenecen a una condición socioeconómica baja, similar a un estudio realizado en Ecuador, que describe que la mayoría de pacientes que desarrollan ND son de condición socioeconómica baja ${ }^{16}$. Esto podría estar relacionado con un mayor riesgo de desarrollar ERC, por dificultad asociada al acceso a la atención de salud por especialista y al cumplimiento de indicaciones médicas.

Dentro de los resultados obtenidos se observa que la mayor parte de la población en estudio pertenece a la atención de Salud Pública, por lo que se infiere que la mayor parte de los usuarios poseen recursos limitados y salarios bajos.

En cuanto al nivel educacional en el estudio el mayor porcentaje de los usuarios refirieron tener entre 9 a 12 años $(38,1 \%)$, seguido por el rango entre 5 a 8 años. Un estudio similar realizado en Brasil detecto un bajo nivel de educación, cuyo resultado se relacionó a las restricciones de acceso a la salud, comprensión de la enfermedad y seguimiento de las indicaciones terapéuticas; lo que podría incidir en el desarrollo y pronóstico de la ND ${ }^{17}$.

Por otro lado, los años con patología diabética previo ingreso a $H D$, se concentraron en el rango de 20 o más años (23,8\%), seguido por el rango de 5 a 9 años (19\%). Una investigación realizada en Venezuela destacó que los pacientes que desarrollaron ND fueron aquellos con menos de 5 años de evolución. Podría asumirse que algunos sujetos tuvieron un mejor control de su DM, presentando ND tardíamente, sin embargo, otros sujetos desarrollaron ND prontamente, pese a que se desconoce si su diagnóstico fue tardío.

En cuanto a la pertenencia etnica por parte de los usuarios, un $73,8 \%$ refirió no pertenecer a ninguna, esto concuerda con un estudio realizado en Ecuador ${ }^{16}$, en el cual se encontró que la ND se presentó mayoritariamente en personas mestizas, en comparación a aquellas pertenecientes a una etnia indígena pura.

Los resultados del test de adherencia a tratamiento, indicaron que la población estudiada fue principalmente no adherente al tratamiento diabético previo al ingreso a HD $(57,1 \%)$, lo que podría indicar un dé- 
ficit de autocuidado que a largo plazo determinaría mayor morbimortalidad. También se debe señalar que un alto porcentaje de los usuarios no fueron derivados a nefrólogo previo a ingreso a HD (71,4\%). Un estudio realizado en Brasil identificó que un $60 \%$ de los usuarios con ERC solo habían recibido evaluación por Nefrólogo al momento de ingresar a HD o solo un mes antes de ingresar a $\mathrm{HD}^{18}$. Esto se relaciona con un tratamiento inadecuado en la etapa previa al ingreso a HD, lo que contribuye a la progresión de la enfermedad. Otro estudio comparativo entre pacientes con seguimiento por nefrólogo menor a 6 meses previos al ingreso a $H D$, con usuarios referidos tempranamente a nefrólogo, encontró que el grupo de usuarios referidos tempranamente con especialista tenían un mejor control metabólico, estancia hospitalaria más corta y mayor porcentaje de casos con acceso vascular permanente al inicio de su $\mathrm{HD}^{19}$, también presentaron mayor tasa de supervivencia. Esto refleja la importancia de la derivación a nefrólogo pues incide directamente en el estado de salud futuro de los usuarios.

Uno de los aspectos a considerar en los resultados de la investigación fue que dos centros de diálisis particulares de la región no aceptaron participar, lo cual incide en la representatividad de los hallazgos obtenidos. Por último, algunos usuarios se negaron a participar lo que redujo el número de la muestra.

Es importante tener en cuenta que son necesarias intervenciones de salud preventivas eficaces y se debe fortalecer la detección y control de los pacientes con DM, para prevenir sus secuelas. En la actualidad la prioridad en el manejo de estos pacientes se ha centrado en el manejo de la etapa final avanzada e irreversible de la enfermedad, con un manejo preventivo muy precario e insuficiente en etapas iniciales, posibles de controlar. Es aquí que el estudio pretende aportar, proporcionando una caracterización de los pacientes con DM que desarrollaron ND y llegaron presentar ERC requirente de $H D$.

Cabe resaltar que más de la mitad de los sujetos de estudio $(71,4 \%)$ refirieron no haber sido derivados a nefrólogo previo a ingresar a HD, indicando que no se pesquiso oportunamente la ERC en etapas iniciales, no se realizó la adecuada derivación y el usuario finalmente no conto con el manejo profesional adecuado. Es fundamental que se fortalezca la evaluación por especialista, pues con un adecuado tratamiento un paciente con ND podría nunca llegar a requerir de HD.
Con respecto a la adherencia al tratamiento, más de la mitad de los usuarios con DM en HD $(57,1 \%)$ fueron no adherentes al tratamiento, esto se interpreto como una deficiencia respecto al autocuidado previo desarrollar ND. Se sugiere fortalecer la labor educativa del profesional de enfermería, específicamente en el fomento del autocuidado, considerando que para realizar una adecuada educación es fundamental conocer las características de la población objetivo, permitiendo planificar estrategias educativas acordes al grupo a educar y que los contenidos logren ser transmitidos. Es por esto que se espera que el presente trabajo contribuya con nuevos conocimientos al quehacer de enfermería y además sirva para entregar un mejor cuidado a los pacientes con DM. Se sugiere complementar los resultados con la realización de un estudio de carácter cualitativo que busque identificar la percepción y experiencias del autocuidado en las personas diabéticas que llegan a presentar ERC en etapa final, buscando comprender mejor su vivencia para proporcionar un mejor cuidado de enfermería.

A la vista de estos resultados podemos concluir que el perfil del paciente diabético que inicia HD de nuestro estudio, son en su mayoría mujeres de edad media, con diagnóstico previo de DM2 sobre la DM1, y con más de 20 años con el diagnóstico de DM. En cuanto al perfil socioeconómico predominan los pacientes con bajos ingresos económicos, estudios básicos y medios. Por último, destacar que presentaron mejor adherencia al tratamiento los hombres respecto a las mujeres.

Recibido: 18-12-19

Revisado: 20-01-20

Modificado: 28-01-20

Aceptado: 10-02-20

\section{Bibliografía}

1. Almaguer Herrera A, Miguel Soca $P$, Reynaldo Será C, Mariño Soler A, Oliveros Guerra R. Actualización sobre diabetes mellitus. Correo Científico Médico [Internet] 2012 [Consultado 5 nov 2017];16(2):[aprox. 0 p.]. Disponible en: http:// revcocmed.sld.cu/index.php/cocmed/article/ view/507. 
2. Ogurtsova K, Fernandes JD, Huang Y, Linnenkamp $U$, Guariguata L, Cho NH, et al. IDF Diabetes Atlas: Global estimates for the prevalence of diabetes for 2015 and 2040. Diabetes Research and Clinical Practice. [Internet] 2017 [Consultado 14 dic 2017]; 128: [aprox. 40-50 p.]. Disponible en: https://doi.org/10.1016/j.diabres.2017.03.024.

3. Deshpande $A D$, Harris-Hayes $M$, Schootman $M$. Epidemiology of diabetes and diabetes-related complications. Phys Ther. 2008;88(11):1254-64.

4. Danaei G, Finucane M M, Y Lu, Singh G.M, Cowan MJ, Paciorek CJ, Et al. National, regional, and global trends in fasting plasma glucose and diabetes prevalence since 1980: systematic analysis of health examination surveys and epidemiological studies with 370 country-years and $2 \cdot 7$ million participants. Lancet 2011;378:31-40.

5. Fabián SMMG, García SFMC, Cobo AC. Prevalencia de síntomas de ansiedad y depresión en pacientes con diabetes mellitus tipo 2 y su asociación con el tipo de tratamiento, complicaciones de la diabetes y comorbilidades. Revista Medicina Interna de Mexico [Internet] 2010 [Consultado 5 nov 2017]; 26 (2) [aprox.100-108 p]. Disponible en: https://www.medigraphic.com/cgi-bin/new/resumen.cgi?IDARTICULO=25297.

6. Zúñiga SM C, Müller H, Flores M. Prevalencia de enfermedad renal crónica en centros urbanos de atención primaria. Rev. méd. Chile 2011; 139(9):1176-84].

7. Gómez- Huelgas R, Martínez Castelao A, Artola $S$, Górriz J, Menéndez E. Documento de Consenso sobre el tratamiento de la diabetes tipo 2 en el paciente con enfermedad renal crónica. Nefrología 2014; 34(1):34-45.

8. Melo A, Mesquita G, Monteiro C. Diagnóstico precoce da doença renal crónica pela estratégica de saúde da família. R Interdiciplinar 2013;6(1):1248.

9. Gorostidi $M$, Santamaría $M$, Alcázar R, Fernández-Fresnedo G, Galcerán J, Goicoechea M, et al. Documento de la Sociedad Española de Nefrología sobre las guías KDIGO para la evaluación y el tratamiento de la enfermedad renal crónica. Nefrología 2014;3(3):273-424.
10. Poblete Badal H. XXXVII CUENTA DE HEMODIÁLISIS CRÓNICA (HDC) EN CHILE 2017. Nefro.cl. [Internet] 2018 [Consultado 27 mar 2018]. Disponible en: http://www.nefro.cl/v2/biblio/registro/23.pdf.

11. Zuñiga C. $30 \%$ del presupuesto AUGE es destinado a financiar la Enfermedad Renal Crónica. Sociedad Chilena de Nefrología. 2014 oct. [Consultado 5 jun 2017] Disponible en: https://www. nefro.cl/v2/post.php?id=272.

12. Schroeder Abreu I, Benedita dos Santos C. Calidad de vida relacionada a la salud de pacientes en hemodiálisis. Rev Enferm UERJ 2013;21(1):95-100.

13. Cuevas Budhart M, Saucedo García R, Romero Quecho G, García Larumbe J, Hernández Paz y Puente A. Relación entre las complicaciones y la calidad de vida del paciente en hemodiálisis. Enferm Nefrol 2017;20(2):112-9.

14. Patologías garantizadas AUGE. Superintendencia de Salud, Gobierno de Chile. [Consultado $16 \mathrm{abr}$ 2018]. Disponible en: http://www.supersalud.gob. cl/664/w3-propertyname-501.html.

15. Palma Campos C. Mujeres con diabetes mellitus e hipertensión, la vivencia de su salud, y el autocuidado a partir de la construcción de la identidad femenina. Rev. cienc. adm. financ. segur. soc;9(2):95-107.

16. Molestina M. Correlación de factores socioeconómicos y metabólicos en la progresión de nefropatía diabética, en pacientes con diabetes mellitus tipo 2, del Hospital Enrique Garcés-Quito, durante los meses de julio a septiembre del año 2014. [Internet]. 2015 [Consultado 27 mar 2018]. Disponible en: http://repositorio.puce.edu.ec/handle/22000/8582.

17. Piccin C, Girardon-Perlini N, de Carli Coppetti L, Hubner da Cruz T, Beuter M, Burg G. Sociodemographic and clinical profile of chronic kidney patients in hemodialysis. Rev Enferm UFPE 2018;12(12):3212-20.

18. Oliveira Junior HM, Claudino Formiga F, Da Silva Alexandre C. Clinical and epidemiological profile of chronic hemodialysis patients in João Pessoa PB. J Bras Nefrol. 2014;36(3):367-74. 
19. Jungers $P$, Massy ZA, Nguyen-khoa T, Choukrou $G$, Robino C, Fakhouri F, et al. Longer duration of predialysis nephrological care is associated with improved long-term survival of dialysis patients. Nephrol Dial Transplant 2001;16(12):2357-64.

20. Ministerio de Salud, Gobierno de Chile. Guía Clínica Insuficiencia Renal Crónica Terminal. 1st Ed. Santiago: Minsal; 2005. p. 10-35.

Este artículo se distribuye bajo una Licencia Creative Commons Atribución-NoComercial 4.0 Internacional. https://creativecommons.org/licenses/by-nc/4.0/

\section{Open Access (c) (7) (\$)}

